\title{
VI. Le Sage's theory of gravitation
}

\author{
James Croll LL.D. F.R.S.
}

To cite this article: James Croll LL.D. F.R.S. (1878) VI. Le Sage's theory of gravitation , Philosophical Magazine Series 5, 5:28, 45-46, DOI: 10.1080/14786447808639382

To link to this article: http://dx.doi.org/10.1080/14786447808639382

$$
\text { 曲 Published online: } 13 \text { May } 2009 .
$$

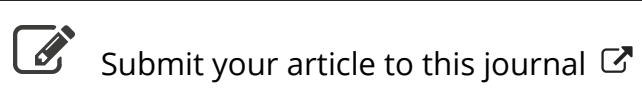

\footnotetext{
Џll Article views: 3
}

Q View related articles $\sqsubset$ 
and he has not considered the propagation of electro-magnetic disturbances in a conducting medium. But according to a former paper of ours, on the "Viscosity of Dielectrics," no dielectric can be assumed to be non-conducting, and the charging of any condenser whatever is always accompanied with absorption phenomena; also absorption certainly increases with conductivity.

Hence although, if a method of experimenting were employed in which a water-condenser of great internal resistance were discharged through wires of less and less resistance for shorter and shorter periods of time, the measured specific inductive capacity might get less and less, and gradually approach a value equal to the square of the index of refraction of water for infinitely long luminous waves (the index of refraction for air being called unity), still practically the measured specific inductive capacity can never be even approximately equal to the refractive index of water, since the absorbed charge is immeasurably greater than the surface-charge. We therefore need not expect to find the specific inductive capacity of water in its variations with temperature consistent with Dr. Gladstone's results for index of refraction. When Prof. Clerk Maxwell takes into account conductivity, his equations are not generally integrable; but even if they were they could not deal with the real case, because he leaves absorption quite out of account.

July 30,1877 .

VI. Le Sage's Theory of Gravitation.

By JaMes CRoLl, LL.D., F.R.S.**

TE SAGE'S theory of gravitation is at present exciting a 1 good deal of attention among physicists. This is perhaps to a considerable extent owing to the fact that some of the conditions arbitrarily assumed by Le Sage in his hypothesis have been proved, from the kinetic theory of gases, to follow as necessary consequences.

A clear and able account of this theory has been given by Mr. Preston in the Philosophical Magazine for September and November last. Mr. Preston has endeavoured to answer all the objections which have been urged against the theory $\dagger$. There is one objection, however, which appears to me not to

* Communicated by the Author.

† Mr. W. B. Taylor, in an interesting article on Kinetic Theories of Gravitation, published in the Smithsonian Report for 1876, lays down six fundamental characteristics of gravitation, with which every theory, he says, must agree. Of these six requirements, Le Sage's theory, he maintains, satisfies but two-namely (1) that the direction of gravity is radial 
have been fully met. It is a necessary condition of Le Sage's theory, in order that gravity may be proportional to mass, that the total volume of the free spaces in a substance in the form of interstices between the molecules must be great compared with the total volume of matter contained in the molecules themselves. This condition of free interstices Mr. Preston considers to be satisfied by assuming the molecules to be small relative to their mean distances.

Were we at liberty to make any assumptions we choose in reference to the smallness of the molecules of matter and their distance apart, we might be able to satisfy the conditions of Le Sage's theory as to mass ; but this we are not at liberty to do. Modern physics has enabled us to determine, at least roughly, the size of the ultimate molecules of matter and also their distance apart. This subject has recently been investigated by Sir William Thomson, the details of which will be found in a remarkable paper in 'Nature,' vol. i. p. 551. Sir William says the diameter of the molecule cannot be less than $\frac{1}{500,000,000}$ of a centimetre. The number of molecules in a cubic centimetre of a liquid or a solid may, he says, be from $3 \times 10^{24}$ to $3 \times 10^{26}$. This gives the distance from centre to centre of two consecutive molecules to be from $\frac{1}{140,000,000}$ to $\frac{1}{460,000,000}$ of a centimetre. Now, if we take the mean of

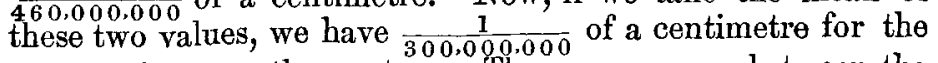
distance between the centres. The mean spaces between the molecules are therefore less than the diameter of the molecules themselves. Under this condition of things, it must be absolutely impossible that a gravific particle, even though it were infinitely small, could penetrate to the extent of a thousandth part of a centimetre into the interior of a body without having its motion stopped by coming into collision with a molecule. Le Sage's theory appears therefore to be utterly irreconcilable with Sir William's conclusions regarding the size of the material molecule. But evon supposing we were to assume, what we are hardly warranted to do, that the molecules are 10,000 times smaller, and their distance apart 10,000 times greater than Sir William Thomson concludes, still this would not assist the theory. The gravific particles would then, no doubt, penetrate a little further into the interior of a body; but beyond a few feet, or perhaps a few inches, no particle could go.

towards the acting mass, and (2) that its intensity diminishes as the square of the distance. But some of Mr. Taylor's objections have already been met by Mr. Preston in his memoir; beside, one or two of Mr. Taylor's fundamental postulates seem doubtful. 